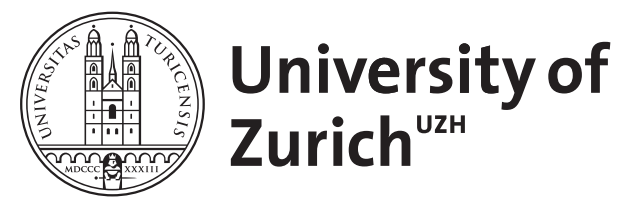

\title{
Maria mediatrix - mittellos mittel aller súnder
}

\author{
Mertens Fleury, Katharina
}

\begin{abstract}
Maria mediatrix represents a paradigm of mediality in the Middle Ages. Mary's power consists in bridging the gap between the celestial sphere and the world, the Christ and mankind. She is full of grace and conveying grace, participating in humanity and divinity by these characteristics. Her influence is based on having given birth to the Christ, accompanied his life until his death on the cross, having suffered and died in her soul with him in love. She incites the individual to follow her on her paths, to love and suffer as she did, to follow the Christ. Her path - if followed - offers the perspective to reach redemption and salvation. But she is also interceding for the sinners. Ascended to heaven and crowned as queen, she is able to change the severity of judgements of her son into mercy. The present article outlines a "mediologic" approach, presenting some developments from the bible to the Middle Ages and giving examples of different ways of Mary's mediation. The latter are mainly focussed on homilies written by Bernhard de Clairvaux (1090-1153) on the occasion of the feasts of the birth and ascension of Mary and five chapters of the, "Büchlein der Ewigen Weisheit" by Heinrich Seuse (1295-1366).
\end{abstract}

DOI: https://doi.org/10.1524/mial.2010.0017

Posted at the Zurich Open Repository and Archive, University of Zurich ZORA URL: https://doi.org/10.5167/uzh-150800

Journal Article

Originally published at:

Mertens Fleury, Katharina (2010). Maria mediatrix - mittellos mittel aller súnder. Das Mittelalter. Perspektiven mediävistischer Forschung, 15(2):33-47.

DOI: https://doi.org/10.1524/mial.2010.0017 


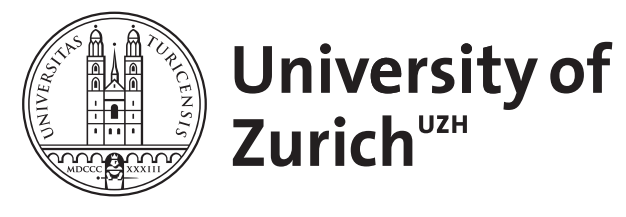

\title{
Maria mediatrix - mittellos mittel aller súnder
}

\author{
Mertens Fleury, Katharina
}

\begin{abstract}
Maria mediatrix represents a paradigm of mediality in the Middle Ages. Mary's power consists in bridging the gap between the celestial sphere and the world, the Christ and mankind. She is full of grace and conveying grace, participating in humanity and divinity by these characteristics. Her influence is based on having given birth to the Christ, accompanied his life until his death on the cross, having suffered and died in her soul with him in love. She incites the individual to follow her on her paths, to love and suffer as she did, to follow the Christ. Her path - if followed - offers the perspective to reach redemption and salvation. But she is also interceding for the sinners. Ascended to heaven and crowned as queen, she is able to change the severity of judgements of her son into mercy. The present article outlines a "mediologic" approach, presenting some developments from the bible to the Middle Ages and giving examples of different ways of Mary's mediation. The latter are mainly focussed on homilies written by Bernhard de Clairvaux (1090-1153) on the occasion of the feasts of the birth and ascension of Mary and five chapters of the, "Büchlein der Ewigen Weisheit" by Heinrich Seuse (1295-1366).
\end{abstract}

DOI: https://doi.org/10.1524/mial.2010.0017

Posted at the Zurich Open Repository and Archive, University of Zurich ZORA URL: https://doi.org/10.5167/uzh-150800

Veröffentlichte Version

Originally published at:

Mertens Fleury, Katharina (2010). Maria mediatrix - mittellos mittel aller súnder. Das Mittelalter. Perspektiven mediävistischer Forschung, 15(2):33-47.

DOI: https://doi.org/10.1524/mial.2010.0017 


\title{
Maria mediatrix - mittellos mittel aller súnder
}

\section{Katharina Mertens Fleury}

\begin{abstract}
Maria mediatrix represents a paradigm of mediality in the Middle Ages. Mary's power consists in bridging the gap between the celestial sphere and the world, the Christ and mankind. She is full of grace and conveying grace, participating in humanity and divinity by these characteristics. Her influence is based on having given birth to the Christ, accompanied his life until his death on the cross, having suffered and died in her soul with him in love. She incites the individual to follow her on her paths, to love and suffer as she did, to follow the Christ. Her path - if followed - offers the perspective to reach redemption and salvation. But she is also interceding for the sinners. Ascended to heaven and crowned as queen, she is able to change the severity of judgements of her son into mercy. The present article outlines a „,mediologic“ approach, presenting some developments from the bible to the Middle Ages and giving examples of different ways of Mary's mediation. The latter are mainly focussed on homilies written by Bernhard de Clairvaux (10901153 ) on the occasion of the feasts of the birth and ascension of Mary and five chapters of the ,Büchlein der Ewigen Weisheit‘ by Heinrich Seuse (1295-1366).
\end{abstract}

Keywords: Maria mediatrix; Mediologie; Bernhard von Clairvaux; Heinrich Seuse

\section{Maria als Weg}

Maria ist mediatrix, denn sie gilt im Mittelalter als barmherzige Gnadenvermittlerin zwischen Gott und den Menschen. Vielfältig sind neben dem Begriff der mediatrix die Bezeichnungen für diese Vermittlungen: Maria ist loesaerinne, süenaerinne, mitlerinne, „Erlöserin, Versöhnerin, Mittlerin“, oder auch advocatrix (Anwältin), adiutrix (Helferin), reparatrix/restauratrix und conciliatrix (Wiederherstellerin/Versöhnerin). ${ }^{1}$ Diese Bezeichnungen erweitern das Bedeutungsspektrum der mediatrix im Hinblick auf verschiedene Funktionen. Daneben ist sie auch soziales Vorbild, sie ist Trägerin von Gnade und verkörpert die Tugenden besonderer Reinheit. Doch besteht das Grundmodell der mediatrix, d. h. (Ver-)Mittlerin, in ihren verschiedenen Vermittlungsleistungen: Die hier angenommene Bedeutung von mediatrix ist deshalb einerseits die der Mitte und des Mittleren zwischen „zwei Momenten“, andererseits die des Prozesses. ${ }^{2}$ Damit ist es möglich, Maria sowohl als

1 Vgl. Wolfgang Beinert, Maria, Mutter Jesu. Historisch-theologisch. In: Lexikon für Theologie und Kirche. 3. Aufl. Bd. 4 (1995), Sp. 1321-1322. Vgl. die Beiträge v. Gerhard Ludwig Müller (Kathol.) u. Norbert Leudemann (Kunstgeschichte), Mittlerin der Gnade. In: Marienlexikon. Bd. 4 (1992), S. $487-$ 493 und Josef Finkenzeller, Miterlöserin. In: Marienlexikon. Bd. 4 (1992), S. 484-486. Siehe zur Terminologie Anselm Salzer, Die Sinnbilder und Beiworte Mariens in der deutschen Literatur und lateinischen Hymnenpoesie des Mittelalters. Mit Berücksichtigung der patristischen Literatur. Eine literarhistorische Studie. Linz 1893 (ND Darmstadt 1967), S. 557-560.

2 Siehe dazu Alexander Roesler, Medienphilosophie und Zeichentheorie. In: Stefan Münker, Alexander Roesler u. Mike Sandbothe (Hgg.), Medienphilosophie. Beiträge zur Erklärung eines Begriffs (Fischer 15757). Frankfurt a. M. 2003, S. 34-52, hier S. 39. Régis Debray verbindet in seiner „Mediologie“ beide, Medium und Prozess der Mediation. Siehe dazu Régis Debray, Einführung in die Mediologie. 
Trägerin des Heils wie als Heilsmittlerin und in dieser Doppelheit als Teil der Heilsökonomie zu betrachten. Untersuchbar ist auf dieser Basis zum einen, wie die Vermittlerin und der durch sie überbrückte Raum zwischen diesen Momenten je konstituiert sind, und zum anderen, wie der Prozess der Vermittlung selbst konturiert ist. ${ }^{3}$ Um solche medialen Konstellationen $\mathrm{zu}$ betrachten, ist es notwendig, historische Zuschreibungsprozesse und Sinnbildungen $\mathrm{zu}$ untersuchen, in denen Maria zur mediatrix wird. ${ }^{4}$ François DAGOGNET führt ein solches Verfahren am Beispiel des Wegs vor. Sein mediologisches Modell ist für mich insofern leitend, als DAGOGNET Medialität nicht nur als zweifache, d. h. von Träger und Prozess definiert, die beide in enger Korrelation stehen (Terrain/Weg und Route bedingen einander), sondern er untersucht auch, wie sich ein bilaterales Muster am Beispiel des Wegs entwickelt (Hin- und Rückweg). Auf Maria mediatrix ist dies übertragbar als Frage nach den Vermittlungsrichtungen in den ausgewählten Dokumenten. Das Modell bietet ferner Ansatzpunkte zur Überlegung, wie die medial produzierte Nähe und Präsenz jeweils ausgestaltet werden und wie weit diese reichen. ${ }^{5}$

Das Modell der Maria mediatrix ist einflussreich, denn Maria hat im Mittelalter durch die Kraft ihrer Symbolik „Glauben, Denken und Fühlen der abendländischen Christenheit maßgeblicher und nachhaltiger geprägt “" als jede andere Frau. ${ }^{6}$ Maria ist im Mittelalter omnipräsent, sei es als Kirchenpatronin, in der Funktion der Hüterin der Gläubigen, als Schutzmantelmadonna oder durch die das Kirchenjahr strukturierenden Marienfeste. Marias Leben schildern Texte und Bilderzyklen: Sie ist dargestellt in der Verkündigung, als Mutter des Jesuskinds im Stall zu Bethlehem, als Begleiterin des Wirkens Jesu und als Betrachterin der Passion Christi oder als Fürbitterin und Himmelskönigin. Maria bewirkt kraft ihrer Heiligkeit Mirakel, sie kehrt aus himmlischen Sphären zu den Menschen zurück, und sie offenbart in ihren Erscheinungen göttliche Geheimnisse. ${ }^{7}$ Sie greift in das Leben der Menschen ein, nicht nur aufgrund ihrer Heiligkeit, sondern auch als weibliches ethisches Vorbild der Nonnen und Jungfrauen wie der Verheirateten und Mütter. ${ }^{8}$

Aus dem Frz. v. Susanne Lötscher (Facetten der Medienkultur). Bern, Stuttgart, Wien 2003, S. 18, $141-$ 148. Überdies leitet er sein Konzept des Medialen von der Christologie ab, geht hier von einer grundsätzlichen Säkularisierung aus. Debrays These, die Mediologie sei nichts anderes als eine verspätete Christologie, lässt die Annahme eines sich in den profanen Bereich erstreckenden Prozesses, einer „Fortsetzung“ und folglich einer Dissemination des Medialen zu. Aber auch in seinen ,inneren“ Logiken ist dieser Begriff im Hinblick auf seinen Gegenstand zu klären. Debray definiert den Doppelkörper des Mediums als eine sowohl passive als auch aktive Funktion des Mediums: „Es ist ein Platz und eine Funktion in einem Beförderungsdispositiv.“ Ebd., S. 149, vgl. S. 151; sein Modell bietet eine Medialität des materiellen Trägers wie des Prozesses der Vermittlung.

3 Oliver Fahle, Zur Einführung. In: Claus Pias, Joseph Vogl u. Lorenz Engell (Hgg.), Kursbuch Medienkultur. Die maßgeblichen Theorien von Brecht bis Baudrillard. 6. Aufl. München 2008, S. 9-10.

4 Vgl. zur Theologie des Neuen Testaments als Sinnbildung Udo Schnelle, Theologie des Neuen Testaments (UTB 2917). Göttingen 2007, S. 16-19 mit weiterer Literatur.

5 Siehe François Dagognet, Route, anti-route, méta-route. In: Les Cahiers de médiologie. Une anthologie. Revue dir. par Régis Debray. Paris 2009, S. 287-291. Siehe in der gleichen Publikation die Beiträge von Daniel Parrochia, Les routes invisibles. Ebd. S. 311-315 sowie Michel Serres, Sortir des réseaux ... Entretien. Ebd. S. 323-327.

6 Vgl. Klaus Schreiner, Maria. Jungfrau, Mutter, Herrscherin (dtv 4707). München, Wien 1994, S. 493.

7 Vgl. Ebd. Einschlägig hierzu ist das Handbuch der Marienkunde. 2. Aufl. 2 Bde. (1996-1997), wie auch das Marienlexikon. 6 Bde. (1988-1994).

8 Siehe dazu Schreiner (Anm. 6). 
Der Titel mediatrix kommt Maria wegen der ihr spezifischen Eigenschaft zu, Gnade zu vermitteln; doch sind in ihre Rolle als Vermittlerin auch soziale und religiöse Ideale eingeschlossen. ${ }^{9}$ Bezüglich dieser Eigenschaft besteht im Mittelalter keine letztgültige Lehrmeinung. Das westliche Christentum kennt bis ins ausgehende Mittelalter hinein zwei Mariendogmen: ${ }^{10}$ den auf dem Konzil von Ephesus im Jahr 431 (DH 252) formulierten Titel der

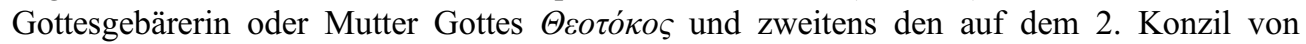
Konstantinopel im Jahr 553 festgelegten Titel der ,immerwährenden Jungfrau' (DH 422). ${ }^{11}$ Die Lateransynode (649) festigt diese Lehrmeinung (DH 503). Erst mit großer zeitlicher Distanz, in Epochen des besonderen Interesses an der Mariologie und der Marienfrömmigkeit, ${ }^{12}$ folgen weitere katholische Dogmen. Sie zeugen jedoch von dem Willen, Diskussionen der Patristik und des Mittelalters zu fixieren und sie weisen deshalb in ihren theologischen Grundlagen in die vorreformatorische Zeit zurück. ${ }^{13}$ So bezieht sich das Dogma der Unbefleckten Empfängnis (1854) in seiner Ausgestaltung auf die Ausführungen zur Heiligung Marias ,De sanctificatione“ in der ,Summa Theologiae“ (STh III, 26) des Thomas von Aquin (um 1225-1274). Im 20. Jahrhundert folgt das Dogma der leiblichen Aufnahme Marias in den Himmel (1950).

Maria mediatrix begegnet nicht in Dogmen, aber relativ spät in der Enzyklika ,Fidentem piumque، (1896). Auch an diesem Rundschreiben wird die bedeutende Nachwirkung der - hier christologisch orientierten - Formulierungen des Thomas von Aquin deutlich (STh II, q. 26a1): Zwar gebe es nur einen Mittler zwischen Gott und den Menschen, den Mensch Christus Jesus (1 Tim 2,5-7), doch könne es daneben auch noch andere Mittler geben. Name und Rolle des Vermittlers gebühren eindeutig Christus, andere fungieren zwar auch als Mittler zwischen Gott und den Menschen, aber nur eingeschränkt, d. h. „,in gewisser Hinsicht" (DH 3320, vgl. auch 4176). ${ }^{14}$ Es sind beispielsweise Engel, Heilige im Himmel, die Propheten und die Priester beider Testamente und in ,höherem Maße“ Ma-

9 Ebd.

10 Der Begriff ist im interkonfessionellen Dialog ambivalent, er ist „ökumenisch verletzend“, so Herbert Vorgrimmler, Neues Theologisches Wörterbuch. 6. Aufl. Freiburg, Basel, Wien 2008, S. 425; die reformatorische Skepsis basiert auf der Zentrierung auf die Schrift und auf Christus und andererseits auf einem definitorischen Problem. Katholischerseits wurde dieser Begriff als Dogma nie verbindlich. Siehe zu diesen Entwicklungen Stefano de Fiores, Maria in der Geschichte von Theologie und Frömmigkeit. In: Handbuch der Marienkunde. 2. Aufl. Bd. 1 (1996), S. 99-266, hier S. 145-162.

11 Siehe Heinrich Denzinger u. Peter Hünermann (Hgg.), Kompendium der Glaubensbekenntnisse und kirchlichen Lehrentscheidungen. 42. Aufl. Freiburg i. Br., Basel, Wien 2009. Im Text zitiert nach den Abschnittnummern und abgekürzt mit DH.

12 Siehe dazu Klaus Schatz, Kirchengeschichte der Neuzeit II (Leitfaden Theologie 20). 3. Aufl. Düsseldorf 1999, S. 57, 131.

13 Sie greifen Diskussionen des Hoch- und Spätmittelalters auf und vollziehen die lehramtliche Fixierung von den Positionen, die sich letztlich durchgesetzt haben. Siehe zur Geschichte das Handbuch der Marienkunde (Anm. 7).

14 Siehe zur christologischen Mediologie Friedrich Schleiermacher, Über die Religion. Reden an die Gebildeten unter ihren Verächtern (RUB 8313). Stuttgart 1969, S. 8 f.; vgl. dazu Christian Kiening, Medialität in mediävistischer Perspektive. Poetica 39 (2007), S. 285-352, hier S. 290-291. Siehe detailliert zur christologischen Mediologie in breitem historischem Überblick den Beitrag von Christian Kiening in diesem Band. Siehe zu den Grundlagen im Alten und Neuen Testament Hans Kessler, Jesus Christus Weg des Lebens. In: Handbuch der Dogmatik. 2. Aufl. Bd. 1. (1995), S. 141-440 mit Lit., bes. S. 251 $260,285$. 
ria. ${ }^{15}$ Diesen Status besitzt sie, weil sie die Mutter des Mittlers ist. Ausführlicher greift dies die während des zweiten vatikanischen Konzils erstellte Konstitution über die Kirche ,Lumen Gentium' auf (1964). ${ }^{16}$ Sie situiert Marias Rolle innerhalb ihrer Mutterschaft in der Gnadenökonomie. Sie fahre auch nach ihrem Tod und ihrer Aufnahme in den Himmel damit fort, uns Gnade zu verschaffen und sie dürfe insofern als Anwältin, Helferin, als Beistand und Mittlerin angerufen werden. ${ }^{17}$ Auch diese Formulierungen greifen auf historische Quellen zurück, d.h. hier auf spätantike Väterzitate. Ohne lineare Entwicklungen der Begriffsverwendung anzunehmen, möchte ich davon ausgehen, dass diese von der Spätantike bis ins 20. Jahrhundert reichenden Traditionen auch Möglichkeiten mittelalterlicher Begriffsverwendungen und Sinnbildungen bezeichnen. ${ }^{18}$ Dann aber ist nach dem Spezifischen des mittelalterlichen Begriffs zu fragen. Möglich ist es, von einem christologischen Standpunkt auszugehen: Wenn einzig Christus, der Mensch und Gottessohn, als universeller Heilsmittler gelten darf und soll, dann sind andere religiöse Mittlerschaften demgegenüber jeweils als Personen mit von Christus abgeleiteten und analogen, aber je spezifischen Mittlerfunktionen abzugrenzen. Von dem im Mittelalter dogmatisch unterdeterminierten Begriff der Maria mediatrix ist nun aber zu erwarten, dass hier das Konzept analoger Vermittlung erweiternde Variationsmöglichkeiten nutzt und bei allem Facettenreichtum konstante Grundlogiken bestehen. ${ }^{19}$

\section{Zwischen Mensch und Christus}

Für die Mariologie und Marias spezifische Medialität sind die Beschreibungen des Neuen Testaments grundlegend; es bietet bereits mediale Grundkonstellationen. Drei Szenen charakterisieren Maria besonders prägnant als Mittlerin: Es ist erstens die Verkündigung an Maria im Lukasevangelium, zweitens die Hochzeit zu Kana und drittens die Kreuzigung im Johannesevangelium. ${ }^{20}$ Das Lukasevangelium erzählt von der Verkündigung des Engels Gabriel an Maria (Lc 1,26-38). Der Engel wendet sich mit dem Gruß an Maria: ave gratia plena Dominus tecum benedicta tu in mulieribus (Lc 1,28) ${ }^{21}$ „Sei gegrüßt gnadenerfüllte, der Herr ist mit dir und du bist gesegnet unter den Frauen.“"Er beruhigt Maria und wiederholt: ne timeas Maria invenisti enim gratiam apud Deum. „Fürchte dich nicht Maria,

15 Siehe Denzinger (Anm. 11), S. 3320.

16 Siehe ,Lumen Gentium` 60 und 62. Denzinger (Anm. 11), 4172-4179.

17 Vgl. Denzinger (Anm. 11), 4177.

18 Siehe ebd. mit Anmerkungen. Es sind Reminiszenzen aus griechischen Kirchenvätern und aus Ambrosius.

19 Aufschlussreich dafür ist die Zusammenstellung im Handbuch von Regina D. Schiewer, Die deutsche Predigt um 1200. Ein Handbuch. Berlin, New York 2008. Sie lässt einen Einblick zu in die Pluralität von Diskursen zu Maria, auch als Fürbitterin und Vermittlerin, im 12. Jh., einer Zeit bedeutender Entwicklungen der Mariologie. Siehe zu Maria mediatrix insbesondere den kunsthistorischen Beitrag von Erwin Panofsky, Imago pietatis. Ein Beitrag zur Typengeschichte des „Schmerzensmanns“ und der „Maria mediatrix“. In: FS für Max Friedländer. Leipzig 1927, S. 261-308 sowie spezifisch zur Fürbitte Marias Catherine Oakes, Ora pro nobis. The Virgin as Intercessor in Medieval Art and Devotion. London 2008.

20 Für die Theologie und die bildende Kunst wird durch die Exegese der Apokalypse auch die Frau mit dem Sternenkranz (Apc 12,1) zu einer Marienfigur und/oder zur ecclesia.

21 Die Bibelzitate folgen der Biblia sacra iuxta vulgatam versionem. Hrsg. v. Roger Gryson u. a.. 4. verb. Aufl. Stuttgart 1994. Die Auslegung dieser Stelle berücksichtigend vgl. auch Georges 2, S. 1738-1739. 
denn du bist begnadet vor Gott.“ (Lc 1,30). Er kündigt ihr die Empfängnis durch den Heiligen Geist und durch die Kraft Gottes an; Maria werde ein Kind empfangen, einen Sohn gebären, der den Namen Jesus und Gottes Sohn tragen soll (Lc 1,35). Marias Reaktion ist zunächst ein Erschrecken, sie ist gerade dadurch in ihrer Menschlichkeit charakterisiert - obwohl sie voll der Gnade und gesegnet ist. In ihr und durch sie verbinden sich so Charakteristika himmlischer und irdischer Sphäre. Und Maria willigt schließlich ein: ecce ancilla Domini fiat mihi secundum verbum tuum. „Siehe ich bin die Magd des Herrn. Es geschehe (an) mir nach deinem Wort" (Lc 1,38). Die Szene ist die Ursprungsszene der Medialität Marias. Der erscheinende Engel ist selbst Bote Gottes und somit ein Mittler, Maria wird in dieser Szene in einen Prozess der Vermittlung eingebunden und selbst zur Mittlerin, d. h. zur künftigen Mutter Christi. Damit sind mediale Verkettungen in dieser Szene bereits angelegt. Es etabliert sich in dieser Szene ein dialogisches Verhältnis, ein Vermittlungsweg, der in zwei Richtungen verläuft. Maria antwortet aktiv auf die Zuwendung Gottes und inseriert sich zugleich als neue Mittlerin in eine Vermittlungsreihe.

Im Johannesevangelium erhält Maria eine besondere Funktion, weil sie ,,inklusionsartig - am Anfang und am Ende des öffentlichen Wirkens Jesu“" auftritt: ${ }^{22}$ Sie ist an der Hochzeit zu Kana in Galiläa (Io 2,1-12) anwesend und gehört bei der Kreuzigung ihres Sohnes (Io 19,25-27) zusammen mit dem Lieblingsjünger Johannes zur präsenten Gemeinde Christi. ${ }^{23}$ In der ersten Szene, an der Hochzeit zu Kana, versiegt der Wein und mit ihm die Festfreude. Maria berichtet das Fehlen des Weins ihrem Sohn. Jesus jedoch zögert zu helfen, denn seine Zeit sei noch nicht gekommen (Io 2,4). Beharrlich weist Maria dennoch die Diener an: Quodcumque dicerit vobis facite! „Was er euch sagt, das tut!“ (Io 2,5). Sie unterstellt sich und andere dem Willen Christi, schürt überdies durch ihre Worte die Erwartungshaltung, dass er doch etwas unternehmen werde. Und Jesus wirkt sein erstes Wunder, er fordert die Diener auf, große Krüge mit Wasser zu füllen, daraus zu schöpfen und den Gastgeber das Getränk kosten zu lassen. Jesus hat, wie sich zeigt, das Wasser in besten Wein gewandelt. Seine Mutter Maria besitzt auch hier die Rolle der mediatrix, nämlich als Bittende für die Festgesellschaft. Sie vermittelt zwischen den Menschen und Christus. Mit ihrer Bitte beginnt zugleich das öffentliche Wunderwirken Jesu.

Im Johannesevangelium ist Maria auch bei der Passion ihres Sohnes anwesend. Sie steht im Johannesevangelium während der Passion in der Nähe des Kreuzes. Sie betrachtet das Sterben ihres Sohnes gemeinsam mit Maria, der Frau des Klopas und Maria Magdalena. Sie ist insofern auch Teil einer Gruppe von Gläubigen, der Anhänger Jesu, d. h. der Kirche in nuce. Christus selbst ist hier Mediator des Heils, am Kreuz wird er zum Erlöser der Menschheit. Seine Mutter Maria hingegen ist hier als Trägerin und künftige Vermittlerin der memoria interpretierbar. Sie repräsentiert die Mutterschaft, erinnert implizit an Vergangenes: die Geburt, die Inkarnation und Jesu erstes Auftreten in der Öffentlichkeit. Zugleich verheißt ihre Präsenz die künftige memoria des hier stattfindenden Heils. Sie ist so Zeugin des Anfangs und des Endes. Durch Maria bieten sich in diesen Beispielen Möglichkeiten der Beziehung zwischen Gott und den Menschen, Christus und der Welt. Sie steht in der Mitte zwischen diesen, durch ihre Vermittlung eröffnet sie neue und z. T. be-

\footnotetext{
22 Siehe Joachim Gnilka, Theologie des Neuen Testaments (Herders theologischer Kommentar zum Neuen Testament. Suppl.-Bd. 5). Freiburg i. Br., Basel, Wien 1994, S. 312.

23 Ebd.
} 
freiende Perspektiven für die Menschen. Die Bibel bietet damit drei Facetten der Vermittlung Marias: Sie ist mediatrix als Gottesgebärerin, Bittende und Zeugin der Passion.

An diesen Modellen arbeitet die im Mittelalter entstehende Theologie und Frömmigkeit weiter. Die biblische Überlieferung zum Leben Marias wurde im Verlauf der Jahrhunderte erweitert. Durch Marienfrömmigkeit geprägte apokryphe Schriften, wie beispielsweise der ,Liber de transitu Mariae' des Ps.-Melito von Sardes (6. Jh. n. Chr.) zur körperlichen Aufnahme Marias in den Himmel, ergänzten die biblischen Vorgaben. ${ }^{24}$ Profilierte schon die Bibel Maria als Fürbitterin bei ihrem Sohn an der Hochzeit zu Kana, so war vor dem Hintergrund des Glaubens an Mariae Himmelfahrt nun die Annahme berechtigt, dass Maria diese Rolle als Fürbitterin auch im Himmel fortsetzen werde. Der Glaube an die himmlische Präsenz der erhöhten Gottesmutter konnte Grund für die Anrufung Marias werden. Zahlreiche Mirakelerzählungen stärkten die Auffassung, dass Maria im Diesseits wie im Jenseits für die Menschen bei Christus vermittelnd eintreten werde. Zudem entwickelte sich ab dem 12. Jahrhundert auch jener Zweig der Passionsliteratur, in dem Maria das Leiden ihres Sohnes klagend wiedererzählt. ${ }^{25}$ Anknüpfungspunkt für mariologische Reflexionen waren bis ins Hochmittelalter hinein besonders häufig die Marienfeste und die sie begleitende Predigt- und Unterweisungsliteratur. Zugleich erhält Maria in dieser Zeit eine besondere Rolle in der Theologie, Liturgie und Frömmigkeit. Das mit ihr verbundene Vermittlungsmodell erhält insofern ebenfalls eine herausragende Bedeutung. ${ }^{26}$

24 Maßgeblich war hier der ,Liber de transitu Mariae‘ des Ps.-Melito von Sardes. Die Legende von den letzten Jahren Marias, ihr Tod und ihre Aufnahme in den Himmel wurden bereits Anfang des 13. Jh. durch Konrad von Heimesfurth in eine deutschsprachige Fassung, die ,Hinvart“ gebracht. Ende des 13. Jh. präsentiert auch die ,Legenda Aurea“ des Jacobus de Voragine diese Erzählung von Mariae Himmelfahrt. Siehe zur Bildtradition auch Frank Büttner u. Andrea Gottdang, Einführung in die Ikonographie. Wege zur Deutung von Bildinhalten. München 2006, S. 72.

25 Vgl. das Handbuch von Tobias Kemper, Die Kreuzigung Christi. Motivgeschichtliche Studien zu lateinischen und deutschen Passionstrakten des Spätmittelalters (MTU 131). Tübingen 2006 und Ulrich Köpf, Die Passion Christi in der lateinischen religiösen und theologischen Literatur des Spätmittelalters. In: Walter Haug u. Burghart Wachinger (Hgg.), Die Passion Christi in Literatur und Kunst des Spätmittelalters (Fortuna Vitrea 12). Tübingen 1993, S. 21-41. Insgesamt weitete sich die Literatur zu Maria stark aus. Siehe dazu beispielsweise Ingeborg Glier, Die deutsche Literatur im späten Mittelalter 12501370. Zweiter Teil. Reimpaargedichte, Drama, Prosa (Geschichte der deutschen Literatur von den Anfängen bis zur Gegenwart 3/2). München 1987, S. 199-203, zu den Marienspielen, die in dieser Untersuchung ausgeklammert bleiben, und zu den Legendensammlungen, die neben anderen Heiligenviten auch Marienlegenden enthalten, siehe S. 306-318.

26 Meine Darlegungen folgen sowohl den bereits aufgezeigten Modellen der Vermittlung wie auch einer chronologischen Gliederung, ohne dabei jedoch von der Annahme unmittelbarer Zusammenhänge oder Abhängigkeiten der Konzepte auszugehen. Kriterium für die Auswahl ist die herausragende Stellung der einzelnen Texte. Siehe zu Maria mediatrix auch die spezifischen Untersuchungen von Marzena Górecka, Das Bild Mariens in der Deutschen Mystik des Mittelalters (Deutsche Literatur von den Anfängen bis 1700). Bern 1999, S. 572-575 und Sandra Fenten, Mystik und Körperlichkeit. Eine komplementär-vergleichende Lektüre von Heinrich Seuses geistlichen Schriften. Würzburg 2007, wie auch Gabriele Lenger, Virgo - Mater - Mediatrix. Untersuchungen zu Priester Wernhers ,Driu liet von der maget‘. Bern, Frankfurt a. M. 1980. 
Maria als Aquädukt

Bernhard von Clairvaux (1090-1153) avancierte im Verlauf des Mittelalters zu einem der einflussreichsten Mariologen. Seine Reputation baut auf einer überschaubaren Zahl von Marientexten auf, jedoch auch auf pseudoepigraphischer und legendarischer Literatur. ${ }^{27}$ Bernhard selbst soll in den Genuss der Milch Marias gekommen sein. ${ }^{28}$

Maria ist für Bernhard Vermittlerin der Gnade, weil sie die Mutter Christi ist. Dies ist die Grundlage von Bernhards Mariologie. Anlass für Bernhards Schriften zu Marienthemen sind liturgische Feste, wie Weihnachten oder Epiphanias. So verfasst Bernhard zu Mariae Himmelfahrt einen Zyklus von sechs Predigten, zu Mariae Verkündigung drei und eine weitere zu Mariae Geburt. Andere Predigten, wie zu Weihnachten oder Pfingsten, wie auch die Hoheliedpredigten schließen Überlegungen zu Maria ein; ferner verfasst Bernhard ein Marienlob. ${ }^{29}$ Als Mutter Christi bietet Maria den Gläubigen einen Königsweg zu Gott. ${ }^{30}$ Entsprechend ist Maria mediatrix auch ein Gegenstand von Bernhards dritter Predigt zur Weihnachtsvigil: Gott hat Maria die Fülle aller Güter in die Hände gelegt und so Christus durch Maria dem Menschen gegeben. Weil der Mensch aber nicht würdig ist, Christus direkt zu empfangen, haben wir ihn durch Maria empfangen. Und Bernhard schließt daraus: Per illam acciperes quidquid haberes. [...] nihil nos Deus habere voluit, quod per Mariae manus non transiret. „Durch sie sollst du empfangen, was immer du hast. [...] Gott wollte uns nichts überlassen als das, was die Hände Marias vermittelten. "31 Maria wird bei Bernhard sogar zum Ursprung allen Heils, da sie die Begegnung der Menschen mit Gott erst ermöglicht hat. Ad illam enim, sicut ad medium, sicut ad arcanum Dei, sicut ad rerum causam. „Auf sie blicken alle wie auf den Mittelpunkt, wie auf das Geheimnis Gottes, wie auf die Ursache der Dinge. “32 Die Unwürdigkeit, Gott direkt zu empfangen, markiert die Grenze zwischen Diesseits und Jenseits. Der Mensch benötigt deshalb Maria als Mittlerin.

Besonders ausführlich breitet Bernhard mediale Logiken im Rahmen der Predigten zu genuinen Marienfesten aus: Mariae Geburt und Himmelfahrt. Mediatrix bedeutet hier, dass sie als die Empfangende und Weitergebende göttlicher Gnade an die Menschen dient. Die Predigt zu Mariae Geburt entfaltet das Thema der Vermittlung sehr breit und verwendet dabei das Mittel der Allegorie. Die medialen Logiken von Gnadenvermittlung und Abbild verklammern sich in der Beschreibung der Verkündigung durch Kausalitäten der Analogie: Wenn der Engel Gabriel bei Maria eintritt und sie grüßt, dann deswegen, weil Maria in ihrer Jungfräulichkeit und Reinheit engelsgleich ist. Marias Mittlerschaft (§ 13, virgine mediante) wird in dieser Predigt jedoch erneut christologisch aufgehoben (Io 1) und erhält ihren Grund im Wirken der göttlichen Personen, d. h. explizit im Zurweltkommen des vom Vater ausgesprochenen Wortes. ${ }^{33}$ Die Mittlerschaft verdeutlichen Präpositionen, wie bei-

27 Dorothee Lauffs u. Norbert Jocher, Bernhard von Clairvaux. In: Marienlexikon. Bd. 1. (1988), S. 477.

28 Siehe zur Ikonographie Christel Squarr, Bernhard von Clairvaux. In: Lexikon der christlichen Ikonographie. Bd. 5 (1973), Sp. 371-385.

29 Vgl. Bernhard von Clairvaux. Sämtliche Werke. Lat./dt. Hg. v. Gerhard B. Winkler. Bd. 1-10. Innsbruck $1990-1999$.

30 Ebd. Bd. 7, S. $77-85$, hier S. 84, $\$ 5$.

31 Ebd. Bd. 7, S. 159-175, hier S. 174, § 9.

32 Ebd. Bd. 8, S. 400-411, bes. S. 404, § 4.

33 In nativitate Beatae Mariae. In: Ebd. Bd. 8, S. 620-647. 
spielsweise querere/habere per Mariam $(\S 7,8)$, ,erbitten/besitzen durch Maria“. Maria ist für die Menschen advocata, „Fürsprecherin“, und der Gläubige ist aufgefordert: ad Mariam recurre (§ 7), ,wende dich an Maria“.

Die Vermittlung ist nach zwei Seiten orientiert: Als jungfräuliche Mutter Jesu erfreut sie den Himmel durch ihre Präsenz, die Erde wiederum ehrt die Erinnerung an sie. Maria ist beides: Mensch und bereits in den Himmel Entrückte. Bernhards Idee der mediatrix materialisiert sich in ihren medialen Prozessen allegorisch: In ihrer Mittlerfunktion ist Maria ein Aquädukt, sie kanalisiert das Wasser des Lebens, den Fluss der Gnade (§ 3). Schon die Väter, Noah und Jakob aber auch Salomo haben an diesem Aquädukt gebaut, doch sprudelte die Gnadenquelle in der Zeit des Alten Testaments in sich selbst (§ 10). Maria löst und öffnet diesen in sich geschlossenen Kreis für die Menschheit als Mittlerin der Gnade (§ 4).

Maria strebt als Aquädukt aktiv nach der Quelle: Sie übersteigt die Menschen und die Engel und reicht zu Gott hinauf durch die Gewalt der Sehnsucht, die Glut der Hingabe, die Reinheit des Gebets, durch die Gerechtigkeit. Ihr Streben ist ein Anklopfen, Bitten und Suchen (§ 5), ein dreifacher Aufstieg im Herzen: durch das Leben, das Gebet und durch den Besuch der Elisabeth im Bergland (§ 9). Dieser Aufstieg führt sie zu Gott, und sie schöpft dort das lebendige Wasser ( $(9)$. Denn Maria ist zwar voll der Gnade, doch reicht ihr dies nicht. Weil sie die Gnade gekostet hat, bittet sie um noch mehr Gnade für die Menschen. Um die Menschheit zu erlösen, vertraut Gott den Preis dafür, Christus, Maria an ( $(6)$. Sie kann daher auch für jene Fürbitte einlegen, die sowohl vor dem Vater als auch vor Christus Angst haben ( $(7)$, und sie können Gnade durch Maria suchen ( $\$ 8)$. Gott, der unzugänglich, unsichtbar und unausdenkbar war, will durch ihr Mitwirken jetzt begriffen, gesehen, gedacht werden (§ 11). Maria nimmt das Wort aus dem Herzen des Vaters selbst auf und bringt es zu den Menschen. Doch soll das Wort nicht leer zurückkehren (§ 13), vielmehr sollen die Ströme der Gnade erneut zu Gott zu gelangen, um noch reichlicher zu fließen (§ 13). Das Wenige, das der Mensch Gott anbieten kann, das solle er Maria anvertrauen, damit sie es Gott darbringe. Gott werde die Opfergabe aus diesen würdigen Händen annehmen $(\S 17-18)$.

Der Text zeigt in enger Verbindung zwei Modelle der Vermittlung. Er präsentiert Marias Aufstiegsmodell als Bedingung einer Partizipation an der Zirkulation der Heilsökonomie. Als Aquädukt ist sie Teil eines „Beförderungsdispositivs“: Maria strebt Gott entgegen, um an das Leben spendende Wasser zu gelangen und es - wenn auch nur tropfenweise an die Menschen zu verteilen. Maria überbrückt so gleich zweimal die Grenze zwischen Immanenz und Transzendenz. Ihr Streben und Bitten durchbricht die vorher in sich geschlossene Zirkulation des göttlichen Gnadenflusses und erschließt die Gnade für alle Menschen. Sie eröffnet so für den Menschen Möglichkeiten des Zugangs zur Transzendenz, befreit ihn aus weltlicher Befangenheit, und sie erlaubt damit auch die „Rückkehr“ des postlapsarischen Menschen zu Christus und zu Gott.

Maria unterscheidet sich in diesen Vermittlungen deutlich von den göttlichen Personen. Zwar kommt allen - Gott, Christus, dem Heiligen Geist und auch Maria - die Eigenschaft zu, Gnade zu spenden. Doch während die göttlichen Personen die Gnade selbst sind, ist Maria das Gefäß der Gnade. Dabei vermag sie es, den Zirkel des göttlichen Gnadenflusses zu durchbrechen und einen Gnadenüberschuss für die Menschen zu erwirken. Sie verteilt diesen Überschuss an Gnade, doch reicht sie die kleinen Gaben der Menschen valorisierend an Gott weiter. Sie produziert dadurch auch in umgekehrter Richtung einen 
Überschuss, was dann die Gnadenströme umso reicher fließen lässt. Die Allegorie des Aquädukts veranschaulicht diese Verbindungen und Vermittlungen, bietet „Bilder“ von der Brücke und Kanalisierung des Wassers des Lebens, d. h. der Gnade. Sie konkretisiert diese formal, wird zum verdeutlichenden Modell. Das bedeutet aber auch, dass dieser Text zur Veranschaulichung seiner Inhalte den vermittelnden „Umweg“ über das Mittel der Allegorie nimmt. Die rhetorischen Logiken sind somit selbst als Sinnüberschuss generierende Vermittlung bewertbar, und es werden insofern in der Konzeption des Textes inhaltliche und kompositorische Vermittlungsleistungen zu einer Einheit zusammengefügt.

\section{Interzessorin}

Marias Fürbittfunktion ist in Bildern und Texten dargestellt worden, ${ }^{34}$ wie im ,Speculum humanae salvationis', das in seiner Konzeption aus dem 13./14. Jahrhundert stammt und das im Spätmittelalter eine sehr große Verbreitung fand. ${ }^{35}$ Das Exemplar von Kremsmünster (Codex Cremifanensis 243) zeigt auf einer Doppelseite Miniaturen von Christus und Maria (fol. 44v und 45r). ${ }^{36}$ Beide machen ihre Verdienste im Hinblick auf die Erlösung der Menschen geltend. Die Darstellungen sind in der Symmetrie, Gestaltung und Farbgebung unübersehbar parallel angelegt. In der linken Miniatur (fol. 44v) zeigt Christus dem in Richterpose innerhalb einer Mandorla thronenden Gottvater seine Wunden bzw. führt die Marterwerkzeuge der Passion vor. Christus steht neben der Geißelsäule, seine Wunden an Händen, Füßen und in seiner Seite sind sichtbar, die Dornenkrone auf seinem Haupt ist blutig rot. Christus erscheint als Mittler, denn im Text ist er als Fürbittender im Jenseits beim Vater charakterisiert: Daz er da für vns bitte sinen vatter. „Dass er dort für uns seinen Vater bitten möge.“ Die Wunden, Zeichen für Christi Erlösungswerk, sind Grund für diese Vermittlung zwischen Menschen und Gott. Die Miniatur auf der gegenüberliegenden Seite (45r) ist nahezu gleich aufgebaut. So steht hier Maria ebenso vor Christus wie der Sohn vor seinem Vater in der linken Miniatur. Maria präsentiert in dieser linken Miniatur ihrem Sohn ihre Brüste, mit denen sie ihren Sohn einst gestillt hat, damit er mit den Sündern Erbarmen habe: daz er sich erbarme vber den sunder. ${ }^{37}$ Auch formal ist der Aufbau gleich. Wie links Gottvater ist rechts Christus in einer Mandorla thronend dargestellt, wie links Christus bittet, so auch Maria rechts. Dieser ähnliche Bildaufbau suggeriert eine analoge Logik: Von links nach rechts gelesen lautet sie: Wie Christus seinen Vater wegen seiner Leiden bitten darf, so bittet auch Maria ihren Sohn Christus. Sie macht ihre Verdienste vor ihrem Sohn geltend und bittet für die Sünder, wie die Inschrift im Bild selbst besagt: orat pro populo. Sie steht als Mensch und barmherzige Mutter zwischen den Sündern und Christus - letzterer wiederum vermittelt zwischen den Menschen und Gottvater.

34 Vgl. Oakes (Anm. 19).

35 Siehe Gunhild Roth u. Marion Grams-Thieme, Speculum humanae salvationis. In: Lexikon des Mittelalters. Bd. 7 (1995), Sp. 2088-2089.

36 Vgl. das Faksimile des ,Speculum humanae salvationis‘. Codex Cremifanensis 243 des Benediktinerstifts Kremsmünster. Komm. v. Willibrord Neumüller (Glanzlichter der Buchkunst 7). Graz 1997.

37 Vgl. Kremsmünster, Benediktinerstift, Codex Cremifanensis 243, fol. 44v und 45r: Das Christusbild trägt die Inschrift: Christus ostendit patri suo cycatrices et vulnera; das Marienbild: Maria ostendit filio suo ubera et orat pro populo. Vgl. auch Darmstadt, HS 2505, fol. 66v und 67r. 
Christus in seiner Strenge und Maria in ihrer Barmherzigkeit können jedoch auch in Opposition stehen: Die Furcht vor Christus leitet dann den Menschen dazu an, sich Maria als Helferin in seiner Not anzuvertrauen. Die ,Wolfenbütteler Marienklage' vertieft diesen Gegensatz der Strenge Christi und Barmherzigkeit Marias in besonders extremer Weise: Maria ist es, die Christus als Gekreuzigten um Barmherzigkeit für die Sünder bittet. Christus weigert sich, Milde walten zu lassen (V. 117-124):

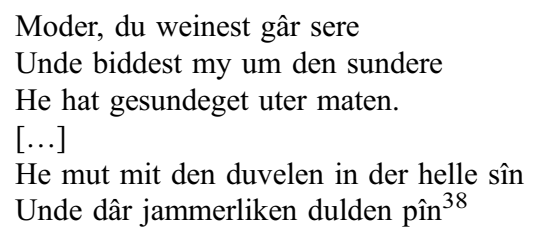

Mutter, du weinst gar sehr und bittest mich für den Sünder, er hat über alle Maßen gesündigt

[...] er muss mit den Teufeln in der Hölle sein. Und dort jämmerliches Leid dulden.

Maria jedoch bittet wegen Mitleidens bei der Kreuzigung ihres Sohnes um Barmherzigkeit für den Sünder (im Allgemeinen). Erst aufgrund ihrer Bitten lässt Christus sich schließlich erweichen und vergibt dem reuigen Sünder die Schuld. In der, Wolfenbütteler Marienklage' wird Maria zu derjenigen, die an der Erlösung und Sündenvergebung der Menschheit motivierend mitwirkt und den strengen, richtenden Christus zur Milde bewegt. Maria bringt hier das Prinzip der Barmherzigkeit in die Heilsgeschichte ein, eröffnet, vermittelt und fördert die Kommunikation zwischen Mensch und Christus.

\section{Mittlerin von Bild- und Abbildrelationen}

Weitaus komplexer ist das dritte Modell der Vermittlung, die Passionsbetrachtung und -wiedergabe wie auch Marias Leidensnachfolge in mittelalterlichen Texten, ausgestaltet. Dies trifft besonders für Maria mediatrix im Werk Heinrich Seuses (1295-1366) zu. ${ }^{39}$ Seuse führt diesen Begriff besonders breit im ,Büchlein der Ewigen Weisheit ${ }^{\star}$ aus. Es ist gerahmt durch eine Kreuzesmeditation und 100 Betrachtungen zur Passion Christi und besteht vor allem aus Dialogen des „Ich“ mit der Ewigen Weisheit. Fünf Kapitel dieses Textes bieten eine dialogisch gestaltete Passionserzählung aus der Perspektive Marias, und damit eine Vermittlung ihres Passionserlebens (Kap. 16-20). ${ }^{40}$ Grundlage dieser Passionserzählung ist der ,(Ps.-)Bernhardstraktat‘, wie Seuse im Prolog zum ,Büchlein der Ewigen

38 Der Sündenfall und Marienklage. Zwei niederdeutsche Schauspiele. Hg. v. Otto Schönemann. Hannover 1855.

39 Siehe dazu bes. Alois M. Haas, Kunst rechter Gelassenheit. Themen und Schwerpunkte von Heinrich Seuses Mystik. Bern, Frankfurt a. M. 1995, bes. S. 125-178.

40 Heinrich Seuse, Deutsche Schriften. Hg. v. Karl Bihlmeyer. Stuttgart 1907 (ND 1961). Ich ziehe das sehr ähnlich konzipierte ,Horologium“ Seuses hier aufgrund der differenzierter zu behandelnden Unterschiede nicht mit ein. Siehe zum Vergleich Fenten (Anm. 26), bes. Kap 1.2.2. Es ist davon auszugehen, dass das ,Büchlein der ewigen Weisheit‘ früher als das ,Horologium“ entstand. Die Unterschiede allerdings sind so erheblich, „dass man nicht von einer bloßen Übersetzung sprechen kann." So Carmen Cardelle de Hartmann, Lateinische Dialoge 1200-1400. Literaturhistorische Studie und Repertorium. Leiden 2007, S. 555 . 
Weisheit ${ }^{\star}$ präzisiert. ${ }^{41}$ Dabei modifiziert Seuse diesen Traktat in signifikanter Weise, indem er den Traktat aufbricht, bedeutend kürzt und in das Gesamtkonzept des ,Büchleins“ integriert. Auch Marias Vermittlungsfunktion, die Seuse besonders in Buch 16 hervorhebt, erscheint hier aus verschiedenen Blickwinkeln: Seuse leitet diesen Teil mit einem Marienlob ein, der Hauptteil ist durch die Marienklage stark geprägt, doch die eigentliche Kreuzigungsszene ist durch einen Dialog zwischen dem diener und Christus ersetzt.

Im Marienlob charakterisiert Seuse Maria mediatrix zunächst. Sie ist mittellos mittel aller súnder „keines Mittlers bedürfendes Mittel aller Sünder“ (263,23-39). Dies charakterisiert sie als Mittlerin, die für die Menschen mittellos anrufbar und erreichbar ist. Im Dialog mit ihr preist das „Ich“ Maria: Weder er, der diener, noch die sündige Seele bedürfe weder des urlobes noch enkeins mitlers gegen dir, ,weder einer Erlaubnis noch eines Mittlers dir gegenüber“" (263,24-25). In dieser Eigenschaft ist sie Fürbitterin (263,31-264,2).

einiger trost aller súndigen herzen, einigú vluht der verschulten menschen, zů der manig nasses ǒge, manig verwundet ellendes herze uf gebotten wird, bis ein gnedigú mitlerin und suenerin entzwúschent mir und der Ewigen Wisheit!

Einziger Trost aller sündigen Herzen, einzige Flucht der in Schuld gefallenen Menschen, an die manch nasses Auge, manch verwundetes leidendes Herz sich wendet. Sei eine gnädige Mittlerin und Sühnerin zwischen mir und der Ewigen Weisheit!

Diese Marienklage in Seuses ,Büchlein der Ewigen Weisheit' basiert auf der Tradition des lateinischen ,(Ps.-)Bernhards'- bzw. ,Quis dabit-Traktats', der auf eine Predigt des Zisterziensers Oglerius von Trino $(1136-1214)$ zurückgeht. ${ }^{42}$ Seuses Text ist m. W. die erste Prosabearbeitung dieser Tradition, und aufgrund des enormen Erfolgs des ,Büchleins der Ewigen Weisheit' erfuhr sie eine weite Verbreitung. ${ }^{43}$ Seuse übernimmt die Tradition des ,(Ps.-)Bernhardstraktats` in weiten Zügen und zitiert diese explizit durch die Übernahme der Anfangs- und Schlüsselworte des Traktats: Wer git minen ǒugen als mengen trehen als mengen bi̊chstaben, daz ich mit liehten trehnen geschribe die ellenden trehen des grundlosen herzleides miner lieben vrǒwen. „Wer gibt meinen Augen so viele Tränen wie Buchstaben, dass ich mit hellen Tränen die Leidenstränen des unmäßigen Herzleides meiner lieben Jungfrau [Maria] aufschreiben kann.“ $(268,6-8) .{ }^{44}$

Diese Verwischung der Ebenen ist ein Prinzip dieser ab dem 13. Jahrhundert sich entwickelnden Texttradition des ,(Ps.-)Bernhardstraktats'. In dieser Tradition stehen Marienklagen in Vers und Prosa wie auch dramatische Fassungen, deren reiches Spektrum in der

41 Vgl. Seuse (Anm. 40), Prolog V. 24-25, S. 197.

42 Vgl. Hans Eggers, Bernhardstraktat. In: Die deutsche Literatur des Mittelalters. Verfasserlexikon. 2. Aufl. Bd. 1. (1978), Sp. 793 f., mit älterer Lit. Zur Datierung siehe Thomas H. Bestul, Texts of the Passion. Latin Devotional Literature and Medieval Society. Philadelphia 1996, S. 13. Vgl. Henri Barré, Le ,Planctus Mariae“ attribué à Saint Bernard. Revue d'ascétique et de mystique 28 (1952), S. 243-266, hier S. 259 .

43 Siehe Alois M. Haas u. Kurt Ruh, Seuse, Heinrich. In: Die deutsche Literatur des Mittelalters. Verfasserlexikon. 2. Aufl. Bd. 8 (1992), Sp. 1109-1129, hier Sp. 1122.

44 Im lateinischen Text: Quis dabit capiti meo aquam et oculis meis ymbrem lacrimarum (§ 1, S. 185), der signifikanterweise auch in den deutschsprachigen Fassungen übernommen wird. Vgl. Gerd Seewald, Die Marienklage im mittellateinischen Schrifttum und in den germanischen Literaturen des Mittelalters. (Diss. masch.) Hamburg 1952, hier zitiert im Wiederabdruck von Edgar Büttner, Die Überlieferung von ,Unser vrouwen klage“ und des ,Spiegel‘ (Erlanger Studien 74). Erlangen 1987, S. $185-199$. 
vorliegenden Untersuchung durch den ,(Ps.-)Bernhardstraktat', ,Unser vrouwen klage“ (UvK) und die ,Bordesholmer Marienklage‘ (BM) vertreten ist. ${ }^{45}$ Maria führt in diesen Texten einschließlich dem ,Büchlein der Ewigen Weisheit' eine Form mystischer Überschreitung von Grenzen in der Rolle der Zeugin und Erzählerin des Passionsgeschehens vor. $^{46}$ Ihr Bericht besteht im Nachvollzug des Kreuzwegs auf den Spuren Christi sowie einer seelischen Passion. Maria erzählt von Christi Verurteilung und Erniedrigung, seiner Kreuztragung und Kreuzigung, Kreuzabnahme und Grablegung. Marias Wiedergabe des Geschehens endet mit der Heimkehr der um Christus Trauernden nach Jerusalem. Die Passion Christi materialisiert sich dabei durch Übertragungen: ${ }^{47}$ Marias Wahrnehmung des Leidens ihres Sohnes schreibt sich in sie ein und prägt sich wie zur sichtbaren Bestätigung dieses inneren Vorgangs auch an ihrem Äußeren aus: Ihr versagt die Stimme ( $\S 12-13$, S. 187-188), sie wird selbst bleich und todesfarben wie ihr Sohn, sie wird leblos, fällt in Ohnmacht. ${ }^{48}$ Als sie das vom Kreuz herabfließende Blut aus Liebe küsst, wird auch sie blutüberströmt. Als Christus am Kreuz mit dem Speer die Seitenwunde erhält (Io 19,34), durchbohrt Maria einmal mehr das Simeonsschwert (vgl. Lc 2,35). Maria wird zum Medium der Passion, sie bildet das Leiden ab und die compassio wird sichtbar. Sie zeigt sich so als Vorbild der Leidenspartizipation. Es entwickelt sich daraus das Potenzial und der Appell zur imitatio. So kontaminiert das Mitleid die Umstehenden. Die an Maria vorgeführten medialen Prozesse der compassio materialisieren sich im Anderen, Nächsten: So fließen auch die Tränen jener, die Maria sehen und das Geschehen begreifen, und entsprechend dieser Forderungen zur Leidensangleichung sollen auch die Rezeption und Niederschrift des Textes unter Tränen stattfinden. Diesen Übertragungsprozess akzentuiert der Traktat ,Unser vrouwen klage‘. Dabei verstärkt sich der dialogische Charakter dieses Vorgangs: Maria empfindet Mitleid mit ihrem Sohn, Christus wiederum hat Mitleid mit ihr (V. 620-625). Der Text appelliert an die Rezipienten (V. 1110-1149 UvK), Marias Zustand zu betrachten, an ihren Schmerz und ihr Leiden zu denken, als sie ihren Sohn sah. Das erzählte Leid - und damit der Inhalt des Buchs - soll sich in das Herz der Rezipienten einschreiben (V. 1163 UvK).

45 Siehe zu dieser Gruppe von Texten Kemper (Anm. 25), S. 64-69.

46 Vgl. Bestul (Anm. 42), S. 165-185. Vgl. auch Kemper (Anm. 25), S. 64. Siehe ausführlich zu den Versfassungen Katharina Mertens Fleury, Klagen unter dem Kreuz: Die Vermittlung von compassio in der Tradition des ,Bernhardstraktats‘. In: Hans-Jochen Schiewer u. Markus Stock (Hgg.), Schmerz in der Literatur des Mittelalters und der Frühen Neuzeit (Transatlantische Studien zu Mittelalter und Früher Neuzeit 4). Göttingen 2010, S. $143-165$.

47 Vgl. zum Definitionsproblem von Mystik Alois M. Haas, Was ist Mystik? In: Kurt Ruh (Hg.), Abendländische Mystik im Mittelalter. Symposion Kloster Engelberg 1984 (Germanistische Symposien-Berichtsbände 7). Stuttgart 1986, S. 319-341, mit Diskussion auf S. 342-346; vgl. auch ders., Mystik als Aussage. Erfahrungs-, Denk- und Redeformen christlicher Mystik (Suhrkamp-Taschenbuch Wissenschaft 1196). Frankfurt a. M. 1996, S. $62-83$ und Otto Langer, Christliche Mystik im Mittelalter. Mystik und Rationalisierung - Stationen eines Konflikts. Darmstadt 2004, S. 41-47.

48 Siehe in der lateinischen Fassung des ,(Ps.-)Bernhardstraktats“ Seewald (Anm. 44), § 7, S. 186; § 9, S. 187; § 23, S. 191; § 40, S. 197 und die Edition beider Texte von Gustav Milchsack, Unser vrouwen klage, in: PBB 5 (1878), S. 193-357. Vgl. dort insbesondere V. 1326-1390. Diese Edition kompiliert die unterschiedlichen Fassungen ,Unser vrouwen klage“ und ,Spiegel', da hier lediglich die Traditionszusammenhänge zur Diskussion stehen, zitiere ich die beiden Fassungen nicht explizit getrennt. 
Diese Subjektivierung wird in einer kurzen Passage von ,Unser vrouwen klage ' besonders deutlich. Dort kippt das Sehen Marias ich sach in das Sehen eines Erzähler-Ichs um (V. 1161,9-11 UvK): ich sihe Jêsum den guoten/erslagen mit den ruoten: / er stât vor mir wunt unt sêr. Subjektivierend konkretisiert und wiederholt sich das Gesehene am „Ich“ abbildhaft als eine Form der Passionsnachfolge. Maria wird so zum „Urbild und Vorbild der Leidensnachfolge Christi““. ${ }^{49}$ Es ist ein Weg, auf dem sich Christus in seiner Passion mitteilt, und es ist der Weg auf dem sich die Menschen mit Christus identifizieren können. Marias Form der Partizipation am Leiden und am Heil, die unio in der Passion ist als imitatio Christi/Mariae bis hin zum innerlichen „Mitsterben“ steigerbar, bis hin zur Grablegung Christi im Herzen. ${ }^{50}$ Doch führt Marias Maßlosigkeit in dieser Haltung auch zu Kritik. So erinnern Christus und auch der Lieblingsjünger Johannes Maria an den Doppelcharakter der Passion: Das Leiden birgt das Heil und die Erlösung der Menschheit. Christus mahnt daher: Marîa, lâ dîn trûren sîn, „Maria lass dein Trauern“ (V. 875 UvK). In der Bordesholmer Marienklage, die ebenfalls in diesem Traditionszusammenhang steht, wendet sich Maria zwar sogar explizit an das Publikum mit der Mahnung, Christi Leiden und ihr Mitleiden zu betrachten (V. 680-685 BM), doch ruft Johannes sie schließlich dazu auf, ihr Weinen zu lassen angesichts des großen Heils, das angebrochen ist (V. 730 BM).

Seuse zitiert diese Tradition, folgt auch inhaltlich diesen Vorgaben. An entscheidender Stelle hebt er sich jedoch davon ab und nutzt im ,Büchlein der Ewigen Weisheit' noch weit mehr als seine Vorlagen das Prinzip der Weitervermittlung des subjektiven Schmerzes. Dies spiegelt sich im Aufbau dieses Abschnitts. In der Erzählchronologie ist es zunächst Maria, die von ire unsaglichem herzleide, „ihrem unaussprechlichem [sic!] Herzeleid“ $(268,5)$ erzählt. Ich waz mir selber vor tót und lebt in ime, und do mir min schónes liep ertôdet wart, do ertǒde ich do erst genlich, ,ich war mir selber tot und lebte in ihm und da mein Leib gestorben war, da erstarb ich erst vollkommen“ (269,8-10). Maria gibt damit ihre unio-Erfahrung wieder, ihre Einheit mit ihrem Sohn im Sterben, im Tod. Maria ist erschüttert, ihr Inneres stirbt mit Christus mit (269,21-23), physische Phänomene begleiten den inneren Prozess: Ich was herzlos worden, min stimme was mir engangen, ich hate min kraft zemal verlorn. „Ich hatte mein Herz verloren, meine Stimme war mir vergangen, ich hatte meine Kraft auf einmal verloren." (269,25-27). Es ist ein Entzug ihrer/seiner selbst. Sich, do erstarb min herze aber und móchti von dien tǒtwunden, so es enphieng, in tusend stuk sin zersprungen, ,Siehe, da starb mein Herz wieder und wollte von deinen Todeswunden, als es sie empfing in tausend Stücke zerspringen“ $(275,20-22) .{ }^{51}$

Seuse durchbricht jedoch an entscheidender Stelle die Perspektive. Denn nachdem Maria ihr Leid geschildert hat, wird die Ewige Weisheit als Gekreuzigter zum Gesprächspartner des dieners. ${ }^{52}$ Nachdem Maria ihre compassio geschildert, erzählt und vorgeführt hat, fordert dann auch der Gekreuzigte den diener zur Kreuzesbetrachtung auf. Die Ewige

49 Vgl. Fenten (Anm. 26), S. 55. Siehe auch Górecka (Anm. 26), S. 466; vgl. Markus Enders, Das mystische Wissen bei Heinrich Seuse (Veröffentlichungen des Grabmann-Institutes zur Erforschung der Mittelalterlichen Theologie und Philosophie. N.F. 37). Paderborn u. a. 1993, S. 288.

50 Siehe die Beispiele bei Górecka (Anm. 26), S. 595.

51 Vgl. ,Unser vrouwen klage‘, V. 670-683. Dieser Topos des zerspringenden Herzens ist in der Tradition des ,Quis dabit'-Traktats lediglich in den Worten Marias präsent und wird hier erstmals - wenn auch intradiegetisch - auf das gläubige Subjekt übertragen.

52 Vgl. Górecka (Anm. 26), S. 600-616. 
Weisheit appelliert an den diener, selbst vor dem Kreuz zu stehen und seinen Blick darauf zu heften: Du solt min trostloses krúz vúr dinú ógen stellen, und solt dir min bitteren marter ze herzen laen gan und alles din liden dar nach bilden. „Du sollst dir mein trostloses Kreuz vor Augen stellen und sollst dir meine bitteren Leiden zu Herzen gehen lassen und all dein Leiden danach bilden." (273,29-31). Der diener soll wie Christus das trostlose lidenne (237,31), die Verlassenheit am Kreuz, nachvollziehen, elend die Verlassenheitsklage rufen $(274,2)$. Sein Leiden soll außen bitter und innen umso gelassener sein, damit der diener Jesus gleich werde und dem Vater gefallen möge (274,7). Er soll nach der Menschen Heil dürsten, im Leben nach Vollkommenheit streben und in Gehorsamkeit und Demut Christus nachsterben: Sih, so ist din krúz nah minem ellenden krúz gebildet und wirt in ihme adellichen volbraht. „Siehe so ist dein Kreuz nach meinem elenden Kreuz gebildet und wird in ihm in edler Weise vollbracht." (274,16-17). Der Mensch soll sich dann in die Seitenwunde Christi schmiegen, darin wohnen und bleiben. Er wird dort mit dem Wasser des Lebens gereinigt und mit Christi rosenfarbenem Blut verziert und mit Christus in Ewigkeit vereint (274,20-22). Diese Wunde ist Zeichen der von Christus erwirkten Erlösung des Menschen von seiner Schuld. Sie erscheint hier als bereits überbrücktes „Dazwischen“. Zugleich schmilzt die Distanz zu Christus und zur Transzendenz durch das „Hineinschmiegen“ in diese Kluft. Diesmal prägt sich nicht Christus dem Subjekt auf, sondern das Subjekt wird Christus mehr als nahe, gelangt in ihn hinein, „bewohnt" dieses Zeichen der Passion, nimmt seine Form an und füllt es aus. Die Leidenspartizipation an sich wird so zum Medium zwischen Immanenz und Transzendenz. Die Vermittlung ist in Seuses Traktat also auf compassio angelegt, aber im Unterschied zur Tradition verbindet er unmittelbar mit der Leidensnachfolge auch eine maßvolle Regulierung. Die äußere Leidensangleichung hat mit innerem Gleichmut, einer Haltung der Gelassenheit, einherzugehen - selbst im Ausruf der Verlassenheit am Kreuz.

Die Vermittlung wird somit komplex: Das Modell von Maria mediatrix ist durch die zitierte Texttradition als affektive Marienklage, als Paradigma für eine Form der Vermittlung und Übertragung der Passion charakterisiert. Maria ist Erzählerin der Passionserfahrung und zugleich Vorbild für die unio. Ihre imitatio ist nachahmbar. Doch in der Abfolge der Dialogpartner wird durch die Änderungen eine weitere, ganz konkrete Perspektivenübernahme deutlich. In der Chronologie der Dialoge erzählt zunächst Maria in der an die Nachfolge Christi und Mariae appellierende Tradition der Marienklage ihr Erleben der Passion. Das „Ich“ der Erzählung übernimmt eine ihr äquivalente Rolle, indem es selbst in einen Dialog mit dem Gekreuzigten eintritt und so am Moment der Heilsvermittlung Christi partizipiert. Die Vermittlungsinstanz geht damit von der Mittlerin zum „Ich“ über und setzt sich auf diese Weise fort. Im Dialog zwischen dem diener und dem Gekreuzigten wird erneut das Modell der Vermittlung zu einem der Unmittelbarkeit, zur unio. Dabei aber mutiert der diener (wie zuvor Maria) selbst zum Mittler der Passion, was sich in der Verallgemeinerung der Erfahrung auf „den Menschen“ ausdrückt. Die Vermittlung der Unmittelbarkeit kann in diesem Verfahren daher weitere, neue Präsenz und Geltung gewinnen es verkettet sich und zeigt dies auch.

\section{Formen der Vermittlung}

Maria mediatrix überbrückt aktiv und passiv als Medium und Mittlerin die Grenze zwischen Immanenz und Transzendenz. Grundlegend für ihre Medialität ist ihr Status als 
auserwählte Gottesmutter; sie ist daher per se Medium der Inkarnation, Medium göttlicher Offenbarung an die Welt. Aber auch umgekehrt wird Maria für die Menschen ein Weg zu Christus und durch ihn auch zu Gottvater. Der Mensch kann sie unvermittelt um Hilfe anrufen und auch darum bitten, menschliche Gaben gottgefällig zu machen.

Doch es wird gerade durch Marias Vermittlungsleistung deutlich, dass durch die menschliche Sünde eine zu überbrückende Kluft zu den göttlichen Personen sich auftut. Christus als universeller Mittler bewirkt durch seine Passion die Erlösung der Menschheit. Doch verursacht der offensichtliche menschliche Mangel die Notwendigkeit weiterer Vermittlungen, denn die Zuwendung Gottes ist durch die Sünde ein stets unverdientes Geschenk, inkommensurabel, eine Gegengabe des Menschen bleibt stets defizitär. So steht Maria in ihrer Barmherzigkeit als Interzessorin dort dem Menschen bei, wo Christus als strenger Richter konturiert ist. ${ }^{53}$ Sie verhilft dann dem Menschen zu einer Stimme vor Christus, wenn ihm der direkte Zugang zu Christus und seinem Vater zu fehlen scheint.

Maria mediatrix ist Teil der Heilsökonomie, partizipiert daran und generiert diese. Sie bricht so den geschlossenen Zirkel des göttlichen Gnadenflusses zugunsten der Menschen auf und schafft damit Neues. Sie fördert bilateral den Gnadenfluss zwischen Transzendenz und Immanenz. Bei Bernhard streckt sie sich aktiv nach dem Heil aus, sie wirkt als Brücke und Kanal für den Fluss der göttlichen Gnade zu den Menschen. In ihrer Medialität erzeugt sie einen Überschuss: Maria ist voll der Gnade, doch sie verlangt nach noch mehr Gnade für die Menschen. Dieser Überschuss wird zum Grund und zur Möglichkeit für ihre Vermittlungen. Er ist jeweils notwendig, um die Prozesse des Gnadenaustauschs in Gang zu setzen. Maria schafft auf diese Weise die Grundlage für den reditus, die Rückkehr des Menschen zum Heil.

Maria erzählt jedoch auch das Heilsgeschehen, die Passion ihres Sohnes. In dieser Wiedergabe schreitet sie den Kreuzweg im Sinn einer Leidensnachfolge ab, bildet die Passion als Zeichen der unio mit ihrem Sohn körperlich ab. Die Passion schreibt sich in ihren eigenen Körper ein, innerlich wie äußerlich. So kann Maria die Passion auch auf diesem Weg nicht nur verbal, sondern auch zeigend ,nach außen“ an Dritte wieder- und weitergeben. Die von solchen Texten geforderte Rezeption besteht nicht nur in der Wahrnehmung ihrer Erzählung, sondern insbesondere in der Wiederholung des Gezeigten, ihrer imitatio. Es entstehen dadurch Medialitäten, die sich nicht mehr auf den Rahmen der Vermittlung zwischen Gott, Maria und den Menschen beschränken. Die Vermittlerin öffnet geschlossene Kreisläufe für neue Beziehungen, ja für Verkettungen medialer Konstellationen, die ihre Wirksamkeit über sich hinaus erst generieren.

Dr. Katharina Mertens Fleury

Deutsches Seminar der Universität Zürich

Schönberggasse 9

CH - 8001 Zürich

kmertens.fleury@ds.uzh.ch

53 Vgl. zu den Veränderungen Rachel Fulton, From Judgement to Passion. Devotion to Christ and the Virgin Mary, 800-1200. Chichester, New York 2002. Die Auffassung von Christus als Richter ist in Fultons Studie das ältere, archaischere Modell. Die Menschennatur Christi steht im 11. und 12. Jh. im Vordergrund, obwohl die historische Kontinuität der älteren Christologie auch aufgrund der hier vorgestellten Quellen angenommen werden darf. 